\title{
Association between absolute neutrophil count and variation at TCIRG1: the NHLBI Exome Sequencing Project
}

Elisabeth A. Rosenthal ${ }^{1}$, Vahagn Makaryan ${ }^{2}$, Amber A. Burt ${ }^{1}$, David R. Crosslin ${ }^{3}$, Daniel Seung Kim ${ }^{3}$, Joshua D. Smith ${ }^{3}$, Deborah A. Nickerson ${ }^{3}$, Alex P. Reiner ${ }^{4}$, Stephen S. Rich ${ }^{5}$, Rebecca D. Jackson ${ }^{6}$, Santhi K. Ganesh ${ }^{7,8}$, Linda Polfus ${ }^{9}$, Lihong Qi ${ }^{10}$, David C. Dale ${ }^{2}$, UW Center for Mendelian Genomics ${ }^{11,12}$, Gail P. Jarvik ${ }^{1,3}$ on behalf of the NHLBI GO Exome Sequencing Project ${ }^{12}$

1. Division of Medical Genetics, School of Medicine, University of Washington, Seattle, WA, USA

2. Division of General Internal Medicine, School of Medicine, University of Washington, Seattle, WA, USA

3. Department of Genome Sciences, University of Washington, Seattle, WA, USA;

4. Department of Epidemiology, School of Public Health, University of Washington, Seattle, WA, USA

5. Center for Public Health Genomics, School of Medicine, University of Virginia, Charlottesville, VA, USA

6. Division of Endocrinology, Diabetes and Metabolism, Department of Internal Medicine, The Ohio State University, Columbus, OH, USA

7. Department of Human Genetics, University of Michigan Medical School, Ann Arbor, MI, USA

8. Department of Internal Medicine, University of Michigan Medical School, Ann Arbor, MI, USA

9. Human Genetics Center, University of Texas Health Science Center, Houston, TX, USA

10. Division of Biostatistics, Department of Public Health Sciences, School of Medicine, University of California, Davis, CA, USA

11. University of Washington, Seattle, Washington, USA

12. Authorship banner is included in supplemental materials

This is the author manuscript accepted for publication and has undergone full peer review but has not been through the copyediting, typesetting, pagination and proofreading process, which may lead to differences between this version and the Version of Record. Please cite this article as doi: $\underline{10.1002 / g e p i .21976 .}$.

This article is protected by copyright. All rights reserved. 
Corresponding author:

Elisabeth A. Rosenthal

erosen@uw.edu

University of Washington Medical Center

1705 NE Pacific St, Box 357720

Seattle, WA 98195

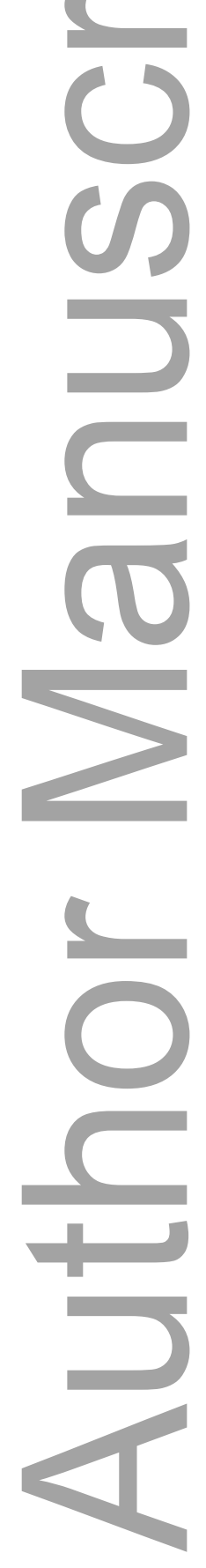

This article is protected by copyright. All rights reserved. 
Abstract

Neutrophils are a key component of innate immunity. Individuals with low neutrophil count are susceptible to frequent infections. Linkage and association between congenital neutropenia and a single rare missense variant in TCIRGl have been reported in a single family. Here, we report on nine rare missense variants at evolutionarily conserved sites in TCIRG1 that are associated with lower absolute neutrophil count (ANC) $(\mathrm{p}=0.005)$ in 1058 participants from three cohorts: the Atherosclerosis Risk in Communities (ARIC), Coronary Artery Risk Development in Young Adults (CARDIA) and Jackson Heart (JHS) studies of the NHLBI Grand Opportunity Exome Sequencing Project (GO ESP). These results validate the effects of TCIRG1 coding variation on ANC and suggest that this gene may be associated with a spectrum of mild to severe effects on ANC.

Key words: Neutropenia, absolute neutrophil count, rare variant replication, next generation sequence data

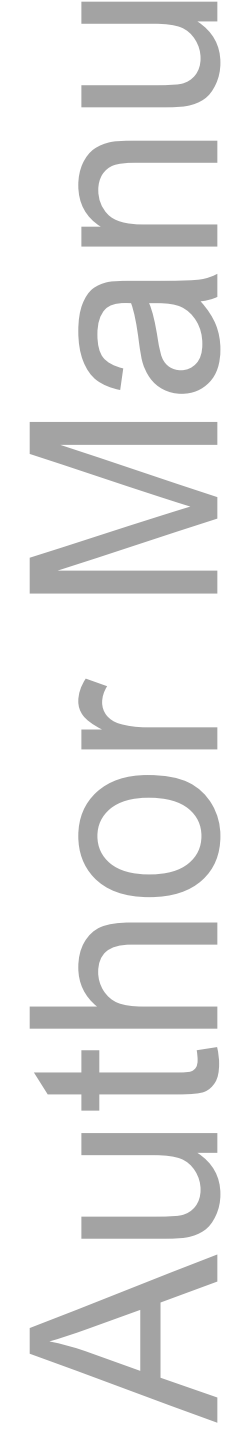

This article is protected by copyright. All rights reserved. 


\section{Introduction}

White blood cells are critical components of innate and adaptive immune responses. About two-thirds of blood leukocytes are neutrophils, the cells which first respond to bacterial or fungal infection. Normal neutrophil counts, in adults, are in the range of $1.8-7.7 \times 10^{9} / \mathrm{L}$ (Dale 2010). Severely neutropenic individuals (neutrophil count $<0.5 \times 10^{9} / \mathrm{L}$ ) suffer from frequent and often severe infections. Severe congenital neutropenia (SCN) (MIM 202700) is characterized by low absolute neutrophil count (ANC) and bacterial infections beginning early in childhood, and is most often inherited as a dominant disorder (Dale and Link 2009, 3-5). However, there are recessive and X-linked forms of congenital neutropenia. Cyclic Neutropenia (CN) (MIM 162800) is an autosomal dominant disease characterized by episodic neutropenia, also beginning in childhood. Variation at ELANE can cause both forms of neutropenia, and variation at other genes (e.g., GFI1, WAS, CXCR4, HAX1, and SDBS) causes SCN (Germeshausen et al. 2013, 905-914; Germeshausen, Ballmaier, and Welte 2007, 93-99; Person et al. 2003, 308-312; Devriendt et al. 2001, 313-317; Ancliff et al. 2006, 21822189; Klein et al. 2007, 86-92; Faiyaz-Ul-Haque et al. 2010, 661-666; Smith et al. 2009, 762770; Germeshausen et al. 2008, 4954-4957; Xia et al. 2009, 535-542; Hauck and Klein 2013, 596-606; Newburger and Dale 2013, 198-206). Individuals of African American (AA) ancestry and some other ethnic groups tend to have lower ANC than individuals with European American (EA) ancestry (Reiner et al. 2011, e1002108). In addition to these known genetic causes, environmental factors correlate with ANC. Medications, radiation therapy, alcohol, and immuno-compromising viruses also affect the ANC.

We previously reported a novel TCIRG1 coding single nucleotide variant (SNV; NG_007878.1:c.2206C>A, p.Arg736Ser, rs587779413) linked and associated with congenital neutropenia (Makaryan et al. 2014, 824-827) (PMID 24753205). Homozygous or compound 
heterozygous mutations in TCIRG1 are also known to cause congenital osteopetrosis, a rare autosomal recessive lethal pediatric disorder (Sobacchi et al. 2013, 522-536). TCIRG1, through alternative splicing, gives rise to two major isoforms, iso-a and iso-b. Iso-a is a fulllength isoform (20 exons) and encodes an a3 subunit of vacuolar H+-ATPase. Iso-b is a shorter isoform, lacking the first five exons of the longer isoform. Iso-a is highly expressed in bone marrow, particularly in osteoclasts (Frattini et al. 2000, 343-346). Iso-b is the major isoform expressed in T-lymphocytes and plays an essential role for T-lymphocyte activation and immune response (Jiang et al. 2013, e58599). Additional splice variants of TCIRG1 with unknown functional effects are expressed in numerous human tissues (heart, liver, kidney, lung, and pancreas) (Susani et al. 2004, 225-235; Smirnova et al. 2005, 943-949).

Rs587779413 is a missense variant in the 18th exon of TCIRG1 which is included in both major isoforms; thus, it is expected to affect both major isoforms.

In this current work, we attempt to validate the association of ANC with rs587779413 and other rare coding variation in TCIRGI in a cohort that is unselected for neutrophil count or infection. We identified rare, conserved coding SNVs in 1058 participants from three cohort studies who had ANC data. In addition to providing replication in an independent dataset, we evaluated evidence for addition functional TCIRG1 SNVs affecting ANC.

\section{Materials}

Phenotype, genotype, and ancestry principal components (PCs) data were collected from the NHLBI Grand Opportunity Exome Sequencing Project (NHLBI GO Exome Sequencing Project (ESP)). Unrelated participants with both percent neutrophils (\%NC) and white blood count (WBC) were included in the study. Permissions to access genotype data was restricted to TCIRG1, DARC SNVs rs2814778 and rs12075, which associate with white blood counts in 
African Americans, and the most common neutropenia pathologic genes ELANE, CSF3R, GFI1, WAS, CXCR4, HAX1, and SDBS(Xia et al. 2009, 535-542; Reiner et al. 2011, e1002108; Crosslin et al. 2012, 639-652).

\section{Methods and Results}

\section{Study Samples}

Three cohort studies collected differential blood counts: the Atherosclerosis Risk in Communities (ARIC) Study, Coronary Artery Risk Development in Young Adults (CARDIA) and Jackson Heart Study (JHS) (Anonymous1989, 687-702; Friedman et al. 1988, 1105-1116; Sempos, Bild, and Manolio 1999, 142-146). These study sites ascertained participants through different vascular phenotypes. The CARDIA study contains a younger cohort, whereas the JHS study includes exclusively AA participants. The demographics and ANC summary data are shown by cohort in Table I.

\section{Adjusted absolute neutrophil count}

Participants with WBC $\geq 11 \times 10^{9} / \mathrm{L}(\mathrm{N}=69)$ were excluded due to possible infection, leaving 3723 participants. Only 381 EA and 343 AA participants were genotyped at rs 2814778 , the DARC SNV which associates with neutrophil count in AA. However, the first 7 ancestry PCs (PC1-7) explained 92\% variation in the rs 2814778 genotype (linear regression F-test $\mathrm{p}<2.2 \mathrm{e}-$ 16) and were used as a proxy for this SNV as well as for ancestry. Only 1060 unrelated participants had both \% NC and WBC. ANC was calculated as \%NC*WBC/100. Of these individuals, 82 had low neutrophil count $\left(\mathrm{ANC}<2 \times 10^{9} / \mathrm{L}\right)$. ANC was square root transformed and then adjusted (ANCadj) for age, sex, PC1-7, study site, and DARC SNV rs 12075 using linear regression (model summarized in Table II). Two participants were removed from the analysis, leaving 1058 participants; one participant was determined to be 
an outlier (Cook's distance for ANCadj was 28 standard deviations from the mean) and one individual had a sex discrepancy; These individuals do not appear in the Tables or Figure.

\section{Genotype Data}

No known pathologic variants at the 7 most common genes (ELANE, CSF3R, GFI1, WAS, $C X C R 4, H A X 1, S D B S)$ associated with neutropenia were found in this data set. Variants in TCIRG1 were assessed for several characteristics which would indicate possible pathology: known to cause autosomal recessive osteopetrosis (ARO), frameshift, early stop, splice, or rare evolutionarily conserved missense, where rare is defined as ESP minor allele frequency $(\mathrm{MAF})<0.005$. Copy number variation was not captured in the available data. Of these, only 9 rare missense variants with the Genomic Evolutionary Rate Profiling score (GERP)>2.95 were found (Cooper et al. 2005, 901-913). These variants, listed in Table III, were considered candidates for causing altered TCIRG1 function, and were identified in 18 participants. All 18 participants were heterozygous for one of the 9 variants. No participant was a homozygote or compound heterozygote for any of these 9 variants. Three variants were carried by multiple (3-5) participants.

\section{Association between TCIRG1 and ANC}

As a group, the 18 participants who were heterozygous for one of these 9 candidate functional variants listed in Table III had lower ANCadj than those who were not (one-sided t-test $\mathrm{p}=0.005)$. These variants explained $0.5 \%$ of the variation in ANCadj. Figure 1 compares the distribution of ANCadj for these 18 heterozygotes to the rest of the participants. Additionally, the mean ANCadj was observed to be lower for each candidate variant. The 6 candidate variants that were each carried by a single participant were observed to be associated with lower ANCadj (between 0.5 and 2.4 standard deviations below the mean) than the 3 variants that were carried by multiple participants (between 0.17 and 0.3 standard 
deviations below the mean). Additionally, 8 individuals without one of these 9 candidate functional SNVs had lower ANCadj (between 2.4 and 3.3 standard deviations below the mean) than the heterozygotes for one of the 9 candidate functional SNVs. All analyses were performed with the statistical software package $\mathrm{R}$ using general linear models(R Core Team 2012).

\section{Discussion}

We recently reported a strong linkage and association of a heterozygous mutation in TCIRG1 and the occurrence of neutropenia in a single large family of European American descent with 11 affected individuals (Makaryan et al. 2014, 824-827). Homozygous mutations in this gene have been shown to be the most common cause for autosomal recessive osteopetrosis, with an estimated frequency of 1 in 250,000 people (Sobacchi et al. 2013, 522-536). We have not found reports indicating that parents of autosomal recessive osteopetrosis patients have been evaluated for neutropenia. We also have not found TCIRG1 mutations in 20 neutropenic patients (all sequenced for most common neutropenia associated genes) enrolled in the severe chronic neutropenia international registry (Dale et al. 2003, 82-93). To further understand TCIRG1 associated neutropenia, we sought to determine if there is a correlation between potentially pathogenic, rare TCIRG1 variants at conserved sites and blood neutrophil count.

The results of this study provide supportive evidence for a role of TCIRG1 variation in determining ANC. Having previously shown that a single TCIRG 1 variant co-segregated and was associated with ANC (Makaryan et al. 2014, 824-827), we now additionally demonstrate an association between rare, coding variants at conserved sites in TCIRG1 and ANC. Notably the more rare of these variants were associated with lower ANC than the ones seen in more than one individual, suggesting a spectrum of effect sizes may be associated with different 
variants. Variation at TCIRGl explains a small portion of the variation in ANC, consistent with the hypothesis that ANC is a complex trait controlled by many biological pathways. Even with this small sample size, the approach of considering only candidate variants in a single gene, reducing multiple testing, allowed detection of statistically significant effects on ANC.

There are several limitations to this study. First, it is possible that other types of variation at TCIRG1 correlate with ANC. There were no TCIRG1 frameshifts, splice variants, or early stops identified in these cohorts; the effects of these more disruptive variants would be of particular interest. Additionally, copy number variation data was not available. It also possible that regulatory variants that are not in the ESP database have an effect. Rare variation at evolutionarily conserved bases is expected to have a larger effect on phenotype than common variation (Gorlov et al. 2008, 100-112). Second, the sample size in our study is under-powered to detect the expected relatively small effects of common variants. However, a larger study would be useful in assessing the correlation between common variants and ANC. Third, the samples were not available to allow Sanger validation. It is unlikely that calling error obscures the results, as exome sequencing of rare variants has been found to be of high quality (Tennessen et al. 2012, 64-69) and misclassification would be expected to reduce power.

The pathological mechanisms for TCIRG1-associated neutropenia are not yet known. This gene encodes multiple proteins affecting hydrogen ion transport and T-cell immune response(Jiang et al. 2013, e58599). In our prior large family with TCIRG1-associated neutropenia, all of the heterozygotes for the pathogenic variant had neutropenia, with variable severity. Some members have severe neutropenia and associated infections whereas others 
are mildly affected. The data in this report suggest that there may be other patients and families with neutropenia attributable to variants in TCIRG1.

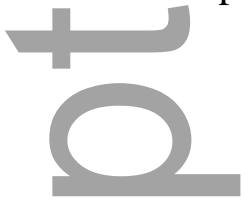

\section{Acknowledgements}

The authors thank UW EMERGE. The authors wish to acknowledge the support of the National Heart, Lung, and Blood Institute (NHLBI) and the contributions of the research institutions, study investigators, field staff and study participants in creating this resource for biomedical research. Funding for GO ESP was provided by NHLBI grants RC2 HL-103010 (HeartGO), RC2 HL-102923 (LungGO) and RC2 HL-102924 (WHISP). The exome sequencing

was performed through NHLBI grants RC2 HL-102925 (BroadGO) and RC2 HL-102926 (SeattleGO). The authors thank the staff and participants of the ARIC, CARDIA and JHS studies for their important contributions.

\section{References}

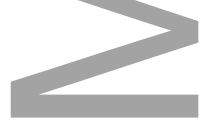

The atherosclerosis risk in communities (ARIC) study: Design and objectives. the ARIC investigators. 1989. American Journal of Epidemiology 129 (4) (Apr): 687-702.

Ancliff, P.J., M. P. Blundell, G. O. Cory, Y. Calle, A. Worth, H. Kempski, S. Burns, et al. 2006. Two novel activating mutations in the wiskott-aldrich syndrome protein result in congenital neutropenia. Blood 108 (7) (Oct 1): 2182-9.

Cooper, G. M., E. A. Stone, G. Asimenos, NISC Comparative Sequencing Program, E. D. Green, S. Batzoglou, and A. Sidow. 2005. Distribution and intensity of constraint in mammalian genomic sequence. Genome Research 15 (7) (Jul): 901-13.

Crosslin, D. R., A. McDavid, N. Weston, S. C. Nelson, X. Zheng, E. Hart, M. de Andrade, et al. 2012. Genetic variants associated with the white blood cell count in 13,923 subjects in the eMERGE network. Human Genetics 131 (4) (Apr): 639-52.

Dale, D. C., T. E. Cottle, C. J. Fier, A. A. Bolyard, M. A. Bonilla, L. A. Boxer, B. Cham, et al. 2003. Severe chronic neutropenia: Treatment and follow-up of patients in the severe chronic neutropenia international registry. American Journal of Hematology 72 (2) (Feb): 82-93. 
Dale, D. C., and D. C. Link. 2009. The many causes of severe congenital neutropenia. The New England Journal of Medicine 360 (1) (Jan 1): 3-5.

Dale, David C. 2010. Chapter 65. neutropenia and neutrophilia. In Williams hematology, 8e., eds. Marshall A. Lichtman, Thomas J. Kipps, Uri Seligsohn, Kenneth Kaushansky and Josef T. Prchal. New York, NY: The McGraw-Hill Companies.

Devriendt, K., A. S. Kim, G. Mathijs, S. G. Frints, M. Schwartz, J. J. Van Den Oord, G. E. Verhoef, et al. 2001. Constitutively activating mutation in WASP causes X-linked severe congenital neutropenia. Nature Genetics 27 (3) (Mar): 313-7.

Faiyaz-Ul-Haque, M., A. Al-Jefri, F. Al-Dayel, J. A. Bhuiyan, H. A. Abalkhail, R. AlNounou, A. Al-Abdullatif, et al. 2010. A novel HAX1 gene mutation in severe congenital neutropenia (SCN) associated with neurological manifestations. European Journal of Pediatrics 169 (6) (Jun): 661-6.

Frattini, A., P. J. Orchard, C. Sobacchi, S. Giliani, M. Abinun, J. P. Mattsson, D. J. Keeling, et al. 2000. Defects in TCIRG1 subunit of the vacuolar proton pump are responsible for a subset of human autosomal recessive osteopetrosis. Nature Genetics 25 (3) (Jul): 343-6.

Friedman, G. D., G. R. Cutter, R. P. Donahue, G. H. Hughes, S. B. Hulley, D. R. Jacobs Jr, K. Liu, and P. J. Savage. 1988. CARDIA: Study design, recruitment, and some characteristics of the examined subjects. Journal of Clinical Epidemiology 41 (11): $1105-16$.

Germeshausen, M., M. Ballmaier, and K. Welte. 2007. Incidence of CSF3R mutations in severe congenital neutropenia and relevance for leukemogenesis: Results of a long-term survey. Blood 109 (1) (Jan 1): 93-9.

Germeshausen, M., S. Deerberg, Y. Peter, C. Reimer, C. P. Kratz, and M. Ballmaier. 2013. The spectrum of ELANE mutations and their implications in severe congenital and cyclic neutropenia. Human Mutation 34 (6) (Jun): 905-14.

Germeshausen, M., M. Grudzien, C. Zeidler, H. Abdollahpour, S. Yetgin, N. Rezaei, M. Ballmaier, B. Grimbacher, K. Welte, and C. Klein. 2008. Novel HAX1 mutations in patients with severe congenital neutropenia reveal isoform-dependent genotypephenotype associations. Blood 111 (10) (May 15): 4954-7.

Gorlov, I. P., O. Y. Gorlova, S. R. Sunyaev, M. R. Spitz, and C. I. Amos. 2008. Shifting paradigm of association studies: Value of rare single-nucleotide polymorphisms. American Journal of Human Genetics 82 (1): 100-12.

Hauck, F., and C. Klein. 2013. Pathogenic mechanisms and clinical implications of congenital neutropenia syndromes. Current Opinion in Allergy and Clinical Immunology 13 (6) (Dec): 596-606.

Jiang, H., W. Chen, G. Zhu, L. Zhang, B. Tucker, L. Hao, S. Feng, et al. 2013. RNAimediated silencing of Atp6i and Atp6i haploinsufficiency prevents both bone loss and inflammation in a mouse model of periodontal disease. PloS One 8 (4): e58599. 
Klein, C., M. Grudzien, G. Appaswamy, M. Germeshausen, I. Sandrock, A. A. Schaffer, C. Rathinam, et al. 2007. HAX1 deficiency causes autosomal recessive severe congenital neutropenia (kostmann disease). Nature Genetics 39 (1) (Jan): 86-92.

Makaryan, V., E. A. Rosenthal, A. A. Bolyard, M. L. Kelley, J. E. Below, M. J. Bamshad, K. M. Bofferding, et al. 2014. TCIRG1-associated congenital neutropenia. Human Mutation 35 (7) (Jul): 824-7.

Newburger, P. E., and D. C. Dale. 2013. Evaluation and management of patients with isolated neutropenia. Seminars in Hematology 50 (3) (Jul): 198-206.

NHLBI GOExome Sequencing Project (ESP). Exome variant server. Available from http://evs.gs.washington.edu/EVS/.

Person, R. E., F. Q. Li, Z. Duan, K. F. Benson, J. Wechsler, H. A. Papadaki, G. Eliopoulos, et al. 2003. Mutations in proto-oncogene GFI1 cause human neutropenia and target ELA2. Nature Genetics 34 (3) (Jul): 308-12.

R Core Team. 2012. R: A language and environment for statistical computing. Vienna, Austria: R Foundation for Statistical Computing.

Reiner, A. P., G. Lettre, M. A. Nalls, S. K. Ganesh, R. Mathias, M. A. Austin, E. Dean, et al. 2011. Genome-wide association study of white blood cell count in 16,388 african americans: The continental origins and genetic epidemiology network (COGENT). PLoS Genetics 7 (6) (Jun): e1002108.

Sempos, C. T., D. E. Bild, and T. A. Manolio. 1999. Overview of the jackson heart study: A study of cardiovascular diseases in african american men and women. The American Journal of the Medical Sciences 317 (3) (Mar): 142-6.

Smirnova, A. S., A. Morgun, N. Shulzhenko, I. D. Silva, and M. Gerbase-DeLima. 2005. Identification of new alternative splice events in the TCIRG1 gene in different human tissues. Biochemical and Biophysical Research Communications 330 (3) (May 13): 9439.

Smith, B. N., P. J. Ancliff, A. Pizzey, A. Khwaja, D. C. Linch, and R. E. Gale. 2009. Homozygous HAX1 mutations in severe congenital neutropenia patients with sporadic disease: A novel mutation in two unrelated british kindreds. British Journal of Haematology 144 (5) (Mar): 762-70.

Sobacchi, C., A. Schulz, F. P. Coxon, A. Villa, and M. H. Helfrich. 2013. Osteopetrosis: Genetics, treatment and new insights into osteoclast function. Nature Reviews.Endocrinology 9 (9) (Sep): 522-36.

Susani, L., A. Pangrazio, C. Sobacchi, A. Taranta, G. Mortier, R. Savarirayan, A. Villa, et al. 2004. TCIRG1-dependent recessive osteopetrosis: Mutation analysis, functional identification of the splicing defects, and in vitro rescue by U1 snRNA. Human Mutation 24 (3) (Sep): 225-35.

Tennessen, J. A., A. W. Bigham, T. D. O'Connor, W. Fu, E. E. Kenny, S. Gravel, S. McGee, et al. 2012. Evolution and functional impact of rare coding variation from deep sequencing of human exomes. Science (New York, N.Y.) 337 (6090) (Jul 6): 64-9. 
Xia, J., A. A. Bolyard, E. Rodger, S. Stein, A. A. Aprikyan, D. C. Dale, and D. C. Link. 2009. Prevalence of mutations in ELANE, GFI1, HAX1, SBDS, WAS and G6PC3 in patients with severe congenital neutropenia. British Journal of Haematology 147 (4) (Nov): 535-42.

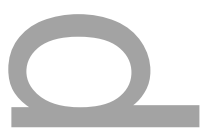

Table I: Demographics and summary neutrophil data, by cohort.

\begin{tabular}{|l|l|l|l|l|l|l|l|l|l|l|}
\hline Site & N & $\%$ Male & \%AA & $\begin{array}{l}\text { Min } \\
\text { Age }\end{array}$ & $\begin{array}{l}\text { Mean } \\
\text { Age }\end{array}$ & $\begin{array}{l}\text { Max } \\
\text { Age }\end{array}$ & $\begin{array}{l}\text { Min } \\
\text { ANC }\end{array}$ & $\begin{array}{l}\text { Mean } \\
\text { ANC }\end{array}$ & $\begin{array}{l}\text { Median } \\
\text { ANC }\end{array}$ & $\begin{array}{l}\text { Max } \\
\text { ANC }\end{array}$ \\
\hline ARIC & 603 & 47 & 46 & 44 & 54.0 & 65 & 0.55 & 3.5 & 3.4 & 8.42 \\
\hline CARDIA & 132 & 58 & 39 & 18 & 26.4 & 33 & 0.69 & 3.5 & 3.3 & 7.17 \\
\hline JHS & 323 & 42 & 100 & 24 & 53.8 & 85 & 0.31 & 3.2 & 2.9 & 8.40 \\
\hline Total & 1058 & 47 & 62 & 18 & 50.5 & 85 & 0.31 & 3.4 & 3.2 & 8.42 \\
\hline
\end{tabular}

Abbreviations: AA, African Ancestry (All other participants are of European ancestry); Min, minimum; Max, maximum; ANC, absolute neutrophil count

Table II: Variables and model from linear regression used to adjust square root of the absolute neutrophil count, before assessing significance of variation at TCIRG1.

\begin{tabular}{|l|l|l|l|}
\hline Covariate & Beta & Std. error & p-value \\
\hline Age & $4.3 \mathrm{e}-03$ & 0.012 & 0.73 \\
\hline Sex(Male) & 0.03 & 0.21 & 0.90 \\
\hline rs12075 & -0.34 & 0.2 & 0.09 \\
\hline PC1-7 & NA & NA & $<2 \mathrm{e}-16$ \\
\hline Site & NA & NA & 0.01 \\
\hline
\end{tabular}

Abbreviations: PC1-7, Ancestry Principal Components 1 through 7

This article is protected by copyright. All rights reserved. 
Table III: The 9 coding variants with GERP $>2.95$ and MAF $<0.005$ considered in the analyses.

\begin{tabular}{|l|l|l|l|l|l|l|l|l|l|l|}
\hline $\begin{array}{l}\text { GRCh37.hg19 } \\
\text { Position on } \\
\text { Chr. 11 }\end{array}$ & $\begin{array}{l}\text { EXON } \\
\text { (of20) }\end{array}$ & RSID & GERP & $\begin{array}{l}\text { MAF\% } \\
\text { EA }\end{array}$ & $\begin{array}{l}\text { MAF\% } \\
\text { AA }\end{array}$ & $\begin{array}{l}\text { MAF\% } \\
\text { ALL }\end{array}$ & $\begin{array}{l}\text { N } \\
\text { EA }\end{array}$ & $\begin{array}{l}\text { N } \\
\text { AA }\end{array}$ & $\begin{array}{l}\text { N } \\
\text { ALL }\end{array}$ & $\begin{array}{l}\text { Mean } \\
\text { ANCadj }\end{array}$ \\
\hline 67809250 & 3 & rs370319355 & 3.72 & 0 & 0.02 & 0.008 & 0 & 1 & 1 & -3.1 \\
\hline 67810304 & 4 & rs377377656 & 4.93 & 0 & 0.03 & 0.009 & 0 & 1 & 1 & -7.8 \\
\hline 67810477 & 5 & rs34227834 & 3.46 & 0.11 & 0.05 & 0.09 & 1 & 0 & 1 & -5.5 \\
\hline 67810963 & 6 & rs372826788 & 3.84 & 0.04 & 0 & 0.02 & 1 & 0 & 1 & -2.3 \\
\hline 67811114 & 7 & rs116001129 & 3.85 & 0 & 0.16 & 0.05 & 0 & 1 & 1 & -4.8 \\
\hline 67814943 & 11 & rs140191063 & 4.07 & 0.10 & 0.09 & 0.10 & 2 & 1 & 3 & -1.0 \\
\hline 67814983 & 11 & rs140963213 & 4.07 & 0.49 & 0.11 & 0.36 & 4 & 1 & 5 & -0.57 \\
\hline 67816589 & 15 & rs115854062 & 4.53 & 0.01 & 0.16 & 0.06 & 0 & 3 & 4 & -0.70 \\
\hline 67817635 & 18 & rs141095902 & 3.89 & 0.01 & 0 & 0.008 & 1 & 0 & 1 & -1.7 \\
\hline Overall & NA & NA & NA & 1.35 & 0.69 & 1.13 & 12 & 8 & 21 & -1.9 \\
\hline
\end{tabular}

$\dagger$ : This SNV may affect splicing, $\$$ : One participant is of indeterminate ancestry

Minor allele frequency (MAF) is from the entire exome sequence project. Abbreviations: GERP, Genomic Evolutionary Rate Profiling score; EA, European Ancestry; AA, African Ancestry; N, count of participants heterozygote for the variant; ANCadj, ANC adjusted for covariates.

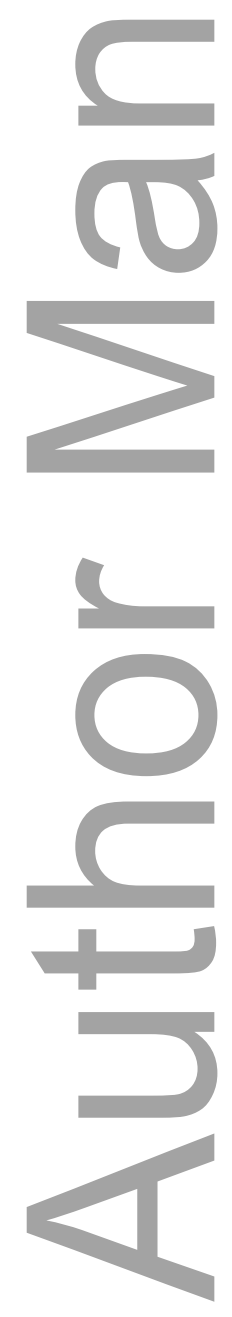

This article is protected by copyright. All rights reserved. 


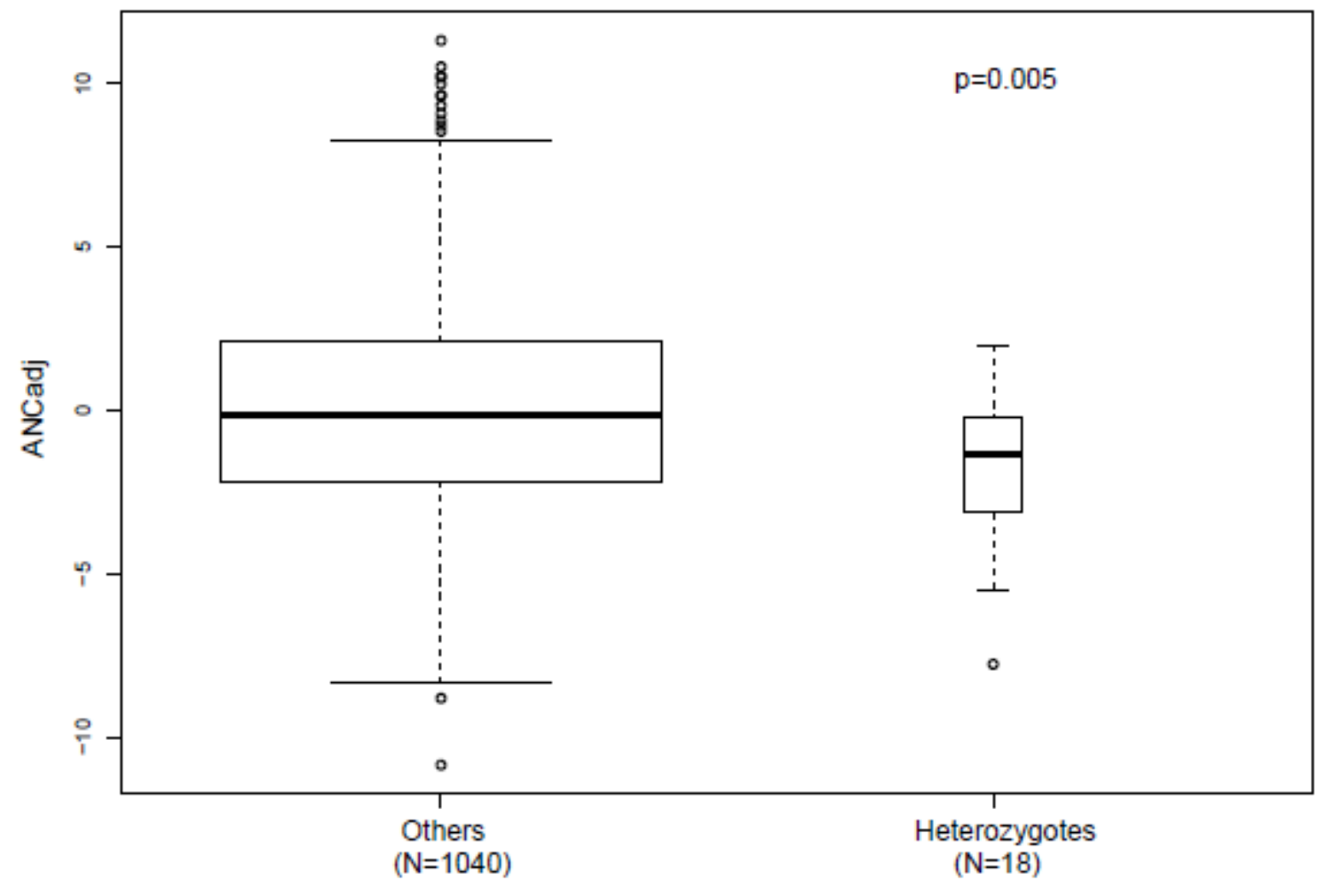

This article is protected by copyright. All rights reserved. 